GENETIC TESTING

Volume 9, Number 3, 2005

(C) Mary Ann Liebert, Inc.

\title{
Accurate Detection and Quantitation of Heteroplasmic Mitochondrial Point Mutations by Pyrosequencing
}

\author{
HELEN E. WHITE, ${ }^{1}$ VICTORIA J. DURSTON,${ }^{1}$ ANNEKE SELLER,${ }^{2}$ CARL FRATTER,${ }^{2}$ \\ JOHN F. HARVEY, ${ }^{1}$ and NICHOLAS C.P. CROSS ${ }^{1}$
}

\begin{abstract}
Disease-causing mutations in mitochondrial DNA (mtDNA) are typically heteroplasmic and therefore interpretation of genetic tests for mitochondrial disorders can be problematic. Detection of low level heteroplasmy is technically demanding and it is often difficult to discriminate between the absence of a mutation or the failure of a technique to detect the mutation in a particular tissue. The reliable measurement of heteroplasmy in different tissues may help identify individuals who are at risk of developing specific complications and allow improved prognostic advice for patients and family members. We have evaluated Pyrosequencing technology for the detection and estimation of heteroplasmy for six mitochondrial point mutations associated with the following diseases: Leber's hereditary optical neuropathy (LHON), G3460A, G11778A, and T14484C; mitochondrial encephalopathy with lactic acidosis and stroke-like episodes (MELAS), A3243G; myoclonus epilepsy with ragged red fibers (MERRF), A8344G, and neurogenic muscle weakness, ataxia, and retinitis pigmentosa (NARP)/Leighs: T8993G/C. Results obtained from the Pyrosequencing assays for 50 patients with presumptive mitochondrial disease were compared to those obtained using the commonly used diagnostic technique of polymerase chain reaction (PCR) and restriction enzyme digestion. The Pyrosequencing assays provided accurate genotyping and quantitative determination of mutational load with a sensitivity and specificity of $100 \%$. The MELAS A3243G mutation was detected reliably at a level of $1 \%$ heteroplasmy. We conclude that Pyrosequencing is a rapid and robust method for detecting heteroplasmic mitochondrial point mutations.
\end{abstract}

\section{INTRODUCTION}

$\mathbf{M}$ ITOCHONDRIAL DISEASES are a clinically heterogeneous group of disorders that occur as a result of mutations of nuclear or mitochondrial DNA (mtDNA), leading to dysfunction of the mitochondrial respiratory chain (DiMauro and Schon, 1998). Nuclear DNA defects are inherited in an autosomal dominant or recessive manner and generally present in childhood. However, the transmission of mtDNA is maternal and affected individuals generally present late in childhood or as adults. mtDNA deletions usually occur de novo and cause sporadic disease with no significant risk to other family members but mtDNA point mutations and duplications can be transmitted.

Disease causing mutations in mtDNA, unlike neutral polymorphic nucleotides (Lagerström-Fermér et al., 2001), are typically heteroplasmic with normal and mutant sequences coex- isting in the same cell (Wallace, 1999). This is analogous to the heterozygous state in mendelian genetics but because each cell may contain thousands of copies of the mitochondrial genome the level of heteroplasmy can vary from $1 \%$ to $99 \%$. Furthermore, the level of heteroplasmy can vary between cells and tissues (Macmillan et al., 1993). Hence, a female harboring a mtDNA mutation may transmit a variable amount of mutated mtDNA to her offspring, which can potentially result in considerable clinical variability amongst siblings within the same family. Prenatal and postnatal genetic testing and interpretation for mitochondrial disorders is therefore problematic. Although there is evidence to show that there is a correlation between the level of heteroplasmy and mitochondrial respiratory function in vivo it has been more difficult to demonstrate an association between level of heteroplasmy and clinical phenotype. It seems likely that a minimum critical number of mutated mtDNA molecules must be present before clinical symptoms appear and

\footnotetext{
${ }^{1}$ National Genetics Reference Laboratory (Wessex), Salisbury District Hospital, Odstock, Salisbury, Wiltshire, United Kingdom.

${ }^{2}$ Oxford Medical Genetics Laboratory, The Churchill Hospital, Headington, Oxford, United Kingdom.
} 
that the pathogenic threshold will be lower in tissues that are dependent on oxidative metabolism. The reliable measurement of heteroplasmy of various mutations in different tissues may help identify individuals who are at risk of developing specific complications and allow improved prognostic advice for patients and family members.

For mutations such as the MELAS mutation (A3243G) it is accepted that higher levels of mutated mtDNA are detected in muscle rather than rapidly dividing tissue (e.g., blood). However, most diagnostic laboratories receive samples that can be obtained in a noninvasive manner such as blood, hair, and buccal swabs that may contain a lower level of mutated mtDNA. Therefore, diagnostic techniques must be capable of reliably detecting low levels of heteroplasmy in such samples. A number of established techniques have been used to genotype and quantitate the level of heteroplasmy for a variety of mitochondrial mutations; denaturing gradient gel electrophoresis (Tully et al., 2000), single-stranded conformational polymorphism (Mashima et al., 1995; Tanno et al., 1995), real-time fluorescent polymerase chain reaction (PCR; Szuhai et al., 2001; Bai and Wong, 2004; He et al., 2002), temporal temperature gradient gel electrophoresis (Boles et al., 2001), Invader technology (Mashima et al., 2004), denaturing high-performance liquid chromatography (DHPLC) (Conley et al., 2003), solid-phase minisequencing (Juvonen et al., 1994; Suomalainen and Syvanen, 2000), and PCR-restriction fragment length polymorphism (RFLP); (e.g. Holt et al., 1990). At present, the most commonly used diagnostic technique used in the United Kingdom is PCR-RFLP (either radioactive or fluorescent). Here we describe the use of Pyrosequencing ${ }^{\mathrm{TM}}$ to genotype and estimate the level of heteroplasmy for six mitochondrial point mutations associated with the following diseases: Leber's hereditary optical neuropathy (LHON), G3460A, G11778A, and T14484C; mitochondrial encephalopathy with lactic acidosis and strokelike episodes (MELAS), A3243G; myoclonus epilepsy with ragged red fibers (MERRF), A8344G; and neurogenic muscle weakness, ataxia, and retinitis pigmentosa (NARP/Leighs): T8993G/C.

\section{MATERIALS AND METHODS}

\section{DNA samples and PCR-RFLP analysis}

Total genomic DNA samples from 50 patients ( 25 males, 25 females; 45 extracted from peripheral blood, 5 extracted from muscle) with presumptive mitochondrial disease were initially analyzed by the commonly used diagnostic method of PCR (fluorescent or nonfluorescent) followed by restriction enzyme digestion using the PCR primers listed in Table 1. Amplicons were generated in a $20-\mu 1$ reaction volume with 5 pmol of forward and reverse PCR primers, $0.2 \mathrm{mM}$ dNTPs (Promega, Madison, WI), $1 \mathrm{X}$ Hotstar Buffer with $\mathrm{MgCl}_{2}$ (Qiagen, Valencia, CA), 1 unit Hotstar Taq (QIAGEN) using 10 ng genomic DNA. PCR conditions for all reactions were $94^{\circ} \mathrm{C}$ for $12 \mathrm{~min}$; 30 cycles with denaturation at $94^{\circ} \mathrm{C}$ for $30 \mathrm{sec}$, annealing at $58^{\circ} \mathrm{C}$ for $30 \mathrm{sec}$ and elongation at $72^{\circ} \mathrm{C}$ for $30 \mathrm{sec} ; 1$ cycle at $72^{\circ} \mathrm{C}$ for 10 minutes; and a final hold at $4^{\circ} \mathrm{C}$. Thermocycling was performed using a PTC-0225 DNA Engine Tetrad (MJ Research, Waltham, MA). $5 \mu$ l PCR product was digested with 2 units of the appropriate restriction enzyme according to manufacturer's instructions.

The LHON mutations G3460A, G11778A, and T14484C were analyzed by nonfluorescent PCR and restriction digestion with AcyI (G3460A causes site loss), MaeIII (G11778A causes site gain) and BanI (primer mismatch creates site for T14484C). The MELAS A3243G, MERRF A8344G, and NARP/Leighs T8993G/C were analyzed by fluorescent PCR. To prevent heteroduplex formation and consequent variability in restriction enzyme digestion, fluorescently labeled reverse primer was added after 30 PCR cycles and a single extension reaction was performed. The restriction enzymes used for analysis of these mutations were HpaII (site gain for the T8993G/C mutation), HaeIII (site loss for G8994A polymorphism), BglI (site gain for A8344G), and ApaI (site gain for A3243G). After digestion, fluorescent products were analyzed using an ABI 3100 Genetic Analyser (Applied Biosystems, Foster City, CA) and the level of heteroplasmy determined by comparison of the cleaved and uncleaved peak areas. Nonfluorescent products were analysed using agarose gel electrophoresis.

\section{Pyrosequencing ${ }^{\mathrm{TM}}$ assays}

Pyrosequencing TM technology is a real-time sequencing method for the analysis of short to medium length DNA sequences (Ronaghi et al., 1998). Four enzymes and specific substrates are used to produce light whenever a nucleotide forms a base pair with the complementary base in a DNA template strand. Biotinylated PCR products are converted to singlestranded templates onto which a sequencing primer is annealed. Analysis begins as the enzymes and substrates are dispensed into the reaction, nucleotides are dispensed sequentially and a light signal is detected and the base registered. If the added nucleotide is not complementary to the next base in the template then no light is generated. The genotype of the mtDNA can be determined using the PSQ ${ }^{\mathrm{TM}}$ 96MA System and the percentage of mutated mtDNA present in the sample can be quantified using the allele frequency quantification function of the SNP software (Biotage AB, Uppsala, Sweden).

DNA samples were randomized and reanalyzed in a blinded fashion for all six mutations using Pyrosequencing ${ }^{\mathrm{TM}}$ technology. Interassay and intra-assay variation for each mutation was determined by analyzing 15 normal control samples in 6 independent experiments and mutated samples were analysed in triplicate to determine reproducibility of results.

PCR amplification and clean-up. The sequences of PCR and sequencing primers (Thermo Electron) used for each assay are listed in Table 1. Amplicons were generated in a 50- $\mu 1$ reaction volume with 15 pmol of forward and reverse PCR primers, $0.2 \mathrm{mM}$ dNTPs (Promega), $1.5 \mathrm{mM} \mathrm{MgCl} 2,1 \times$ Buffer II (Applied Biosystems), 1 unit AmpliTaq Gold (Applied Biosystems) using $10 \mathrm{ng}$ genomic DNA. PCR conditions for all reactions were $94^{\circ} \mathrm{C}$ for 7 minutes; 50 cycles with denaturation at $94^{\circ} \mathrm{C}$ for $30 \mathrm{sec}$, annealing at $60^{\circ} \mathrm{C}$ for $30 \mathrm{sec}$ and elongation at $72^{\circ} \mathrm{C}$ for $30 \mathrm{sec} ; 1$ cycle at $72^{\circ} \mathrm{C}$ for $7 \mathrm{~min}$; and a final hold at $4^{\circ} \mathrm{C}$. Thermocycling was performed using a PTC-0225 DNA Engine Tetrad (MJ Research).

Single-stranded biotinylated PCR products were prepared for sequencing using the Pyrosequencing ${ }^{\mathrm{TM}}$ Vacuum Prep Tool. 


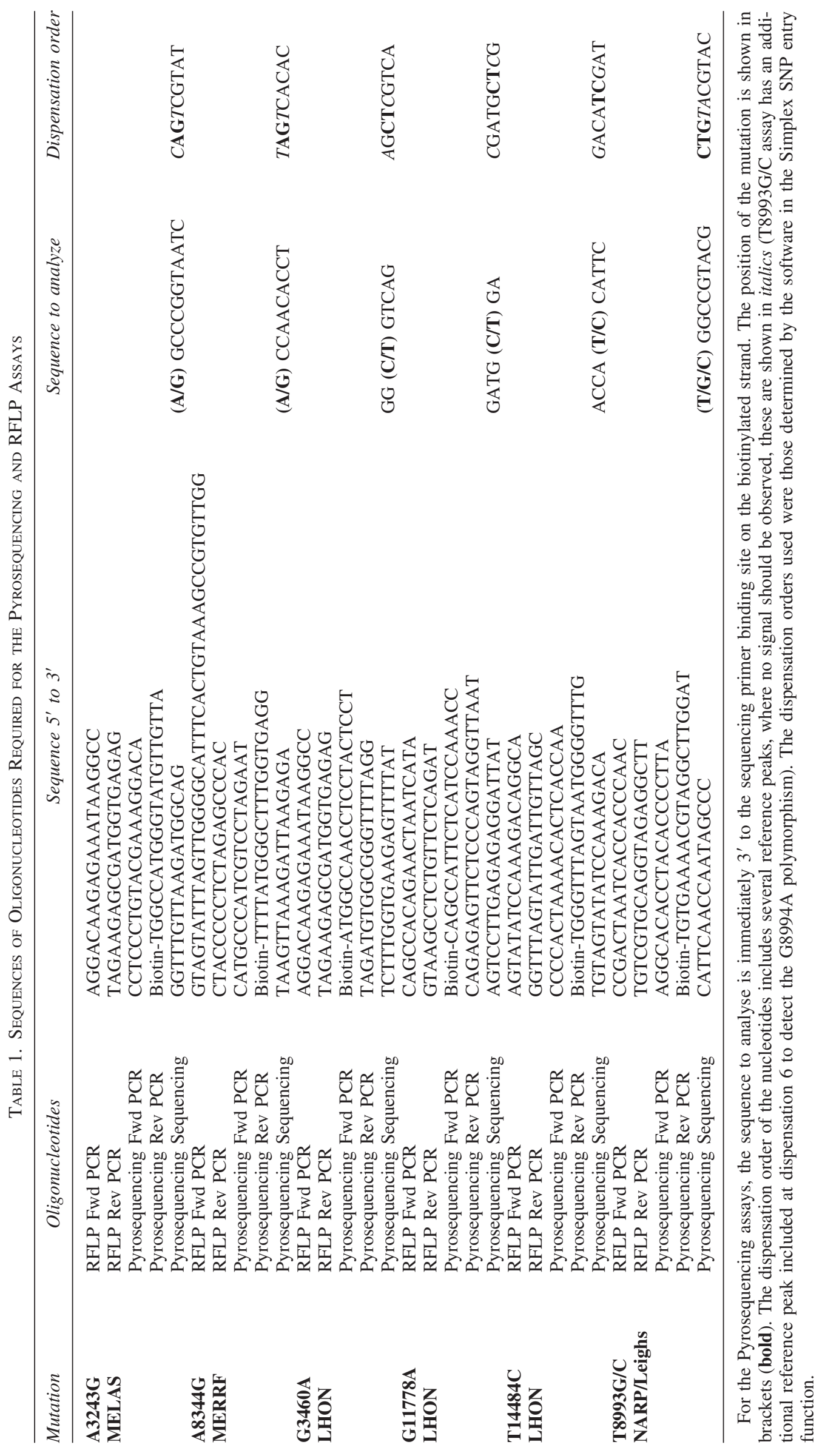


Three microliters of Streptavidin Sepharose ${ }^{\mathrm{TM}} \mathrm{HP}$ (Amersham, Piscataway, NJ) was added to $37 \mu$ l binding buffer $(10 \mathrm{mM}$ Tris- $\mathrm{HCl} \mathrm{pH}$ 7.6, $2 \mathrm{M} \mathrm{NaCl}, 1 \mathrm{mM}$ ethylenediaminetetraacetic acid [EDTA], $0.1 \%$ Tween 20) and mixed with $20 \mu \mathrm{L}$ PCR product and $20 \mu \mathrm{l}$ high purity water for $10 \mathrm{~min}$ at room temperature using a Variomag Monoshaker (Camlab, Cambridge, UK). The beads containing the immobilized templates were captured onto the filter probes after applying the vacuum and then washed with $70 \%$ ethanol for $5 \mathrm{sec}$, denaturation solution $(0.2 \mathrm{M} \mathrm{NaOH})$ for 5 seconds and washing buffer $(10 \mathrm{mM}$ TrisAcetate $\mathrm{pH}$ 7.6) for 5 seconds. The vacuum was then released and the beads released into a PSQ 96 Plate Low containing 45 $\mu \mathrm{l}$ annealing buffer (20 mM Tris-Acetate, $2 \mathrm{mM} \mathrm{MgAc} 2 \mathrm{pH}$ 7.6), $0.3 \mu \mathrm{M}$ sequencing primer. For the $\mathrm{T} 8993 \mathrm{C} / \mathrm{G}$ assay, we found it necessary to add $1 \mu \mathrm{l}$ single stranded binding protein (Promega, $2.2 \mu \mathrm{g} / \mu \mathrm{l}$ ) to eliminate secondary structure in the template DNA. The samples were heated to $80^{\circ} \mathrm{C}$ for $2 \mathrm{~min}$ and then allowed to cool to room temperature.

Pyrosequencing reactions and data analysis. Pyrosequencing reactions were performed according to the manufacturer's instructions using the PSQ 96 SNP Reagent Kit, which contained the enzyme and substrate mixture and nucleotides. Assays were performed using the nucleotide dispensation orders shown in Table 1. The sample genotype and percent heteroplasmy were determined using the allele frequency quantification (AQ) function in the SNP Software (Biotage AB). Samples were considered to have the mutation if the value of percent heteroplasmy was greater than three standard deviations from the mean value obtained from the normal replicates.

\section{Threshold detection of $A 3243 G$ mutation}

DNA samples with known levels of heteroplasmy were prepared to determine the lowest level of the A3243G mutation that could be detected reliably. In 2004, Urata and colleagues analyzed 40 healthy donors and MELAS patients using peptide nucleic acid probe-directed PCR clamping and demonstrated that a mutational load as low as $0.1 \%$ can be clinically significant. Therefore, the detection of low frequency heteroplasmy for this mutation is particularly important. DNA from a MELAS patient was amplified using the primers $5^{\prime}$ tgcagccgctattaaaggtt $3^{\prime}$ and 5' ggttcggttggtctctgcta 3' (amplified region 30143894). The resulting 880-bp amplicon was cloned into the vector pCR2.1 (Invitrogen, Carlsbad, CA) and several colonies were sequenced to identify 2 clones; one with a wild-type genotype and the other with the MELAS mutation. DNA from each clone was quantified and diluted to a final concentration equivalent to $2 \times 10^{4}$ mitochondrial genomes per microliter (diluted in $10 \mathrm{ng}$ salmon sperm DNA). The wild-type and mutated DNA were mixed to generate samples with the A3243G mutation present at levels ranging from 1\%-100\%. Each sample was analyzed in triplicate using the Pyrosequencing TM assay, fluorescent and nonfluorescent PCR-RFLP (using identical primer sets), and the detection threshold for each technique was determined.

\section{RESULTS}

\section{Sensitivity, specificity, and reproducibility}

To determine the background levels for each Pyrosequencing $^{\mathrm{TM}}$ assay, 15 normal DNA samples were analyzed in six independent experiments. The mean and standard deviation values for intra and inter assay analysis are shown in Table 2. No differences were observed between the interassay and intra-assay analysis, which suggests that the background variation seen in these assays is consistent and that the assays are robust. Figure 1 shows the genotype and percent heteroplasmy obtained from the 50 patient DNA samples using the Pyrosequencing assays compared to the results obtained using the routine PCRRFLP method for all mutated samples. Error bars for the Pyrosequencing data indicate the standard deviation for triplicate analysis. Samples were considered to harbor the mutation if the AQ value obtained was greater than three standard deviations

Table 2. Interassay and Intra-Assay Analysis of Fifteen Normal Samples Tested in Six Independent Experiments to Determine Level of Background Detection of Mutant Allele for Each Assay

\begin{tabular}{|c|c|c|c|c|c|}
\hline \multirow[b]{2}{*}{ Mutation } & \multicolumn{2}{|c|}{ Interassay analysis $(\mathrm{n}=15)$} & \multicolumn{2}{|c|}{ Intra-assay analysis $(\mathrm{n}=6)$} & \multirow[b]{2}{*}{ Mean $+3 S D$} \\
\hline & Mean & $\begin{array}{l}\text { Standard } \\
\text { deviation }\end{array}$ & Mean & $\begin{array}{l}\text { Standard } \\
\text { deviation }\end{array}$ & \\
\hline $\begin{array}{l}\text { A3460G } \\
\text { LHON }\end{array}$ & 2.86 & 1.62 & 2.88 & 1.62 & 7.72 \\
\hline $\begin{array}{l}\text { G11778A } \\
\text { LHON }\end{array}$ & 0.05 & 0.14 & 0.06 & 0.22 & 0.72 \\
\hline $\begin{array}{l}\text { T14484C } \\
\text { LHON }\end{array}$ & 2.13 & 0.63 & 2.13 & 0.98 & 5.07 \\
\hline $\begin{array}{l}\text { A3243G } \\
\text { MELAS }\end{array}$ & 0.06 & 0.07 & 0.06 & 0.11 & 0.39 \\
\hline $\begin{array}{l}\text { A8344G } \\
\text { MERRF }\end{array}$ & 0.13 & 0.32 & 0.13 & 0.50 & 1.63 \\
\hline $\begin{array}{l}\text { T8993G/C } \\
\text { NARP/Leighs }\end{array}$ & 0.01 & 0.02 & 0.01 & 0.03 & 0.04 \\
\hline
\end{tabular}

$\mathrm{SD}$, standard deviation. 
A

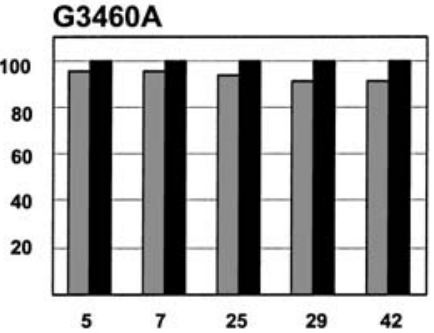

B

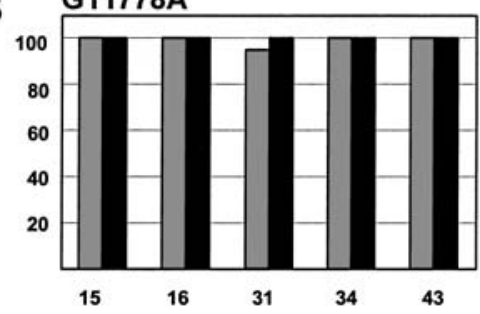

C

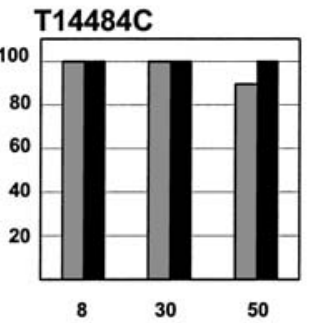

D

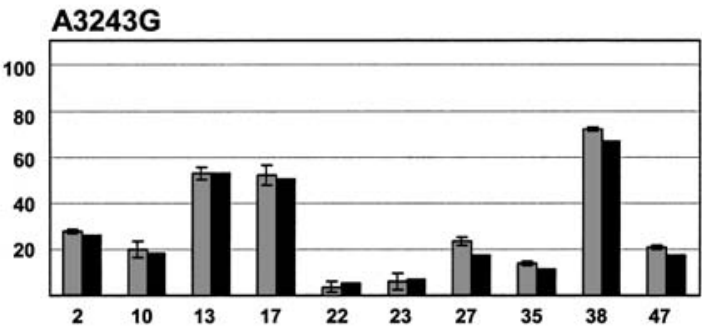

E

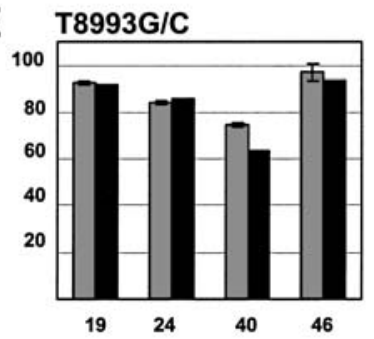

F

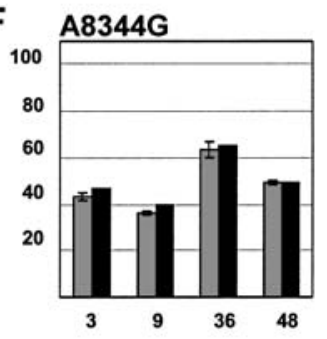

\section{Pyrosequencing}

\section{PCR RFLP}

FIG. 1. Percentage heteroplasmy detected by the Pyrosequencing assays compared to the diagnostic result obtained using nonfluorescent polymerase chain reaction-restriction fragment length polymorphism (PCR-RFLP). A: G3460A. B: G11778A. C: T14484C and fluorescent PCR-RFLP. D: A3243G. E: T8993G/C. F: A8344G. The x axis shows the sample number and the y axis shows the percentage heteroplasmy detected. In graphs (a) to (c) the $100 \%$ heteroplasmy obtained for PCR-RFLP indicates that no wild-type product was viewed on a $3 \%$ agarose gel after restriction enzyme digestion.

from the mean value obtained from the normal replicated samples (Table 2). The genotypes obtained using the Pyrosequencing assays were $100 \%$ concordant with those obtained using PCR-RFLP and the levels of heteroplasmy detected using both techniques were essentially identical. Of the 50 patient DNA samples 13 were found to have 1 of the 3 LHON mutations, 10 had the A3243G MELAS mutation, 4 were positive for the MERRF A8344G mutation, 4 carried the NARP/Leighs T8993G/C mutation, and no mutation was detected in 19 samples. None of the samples had the G8994A polymorphism. The Pyrosequencing assays were $100 \%$ sensitive and $100 \%$ specific taking into account the respective background values. The determination of the level of heteroplasmy using the Pyrosequencing assays was highly reproducible. For the mutated samples analyzed in triplicate, no deviation from the mean was observed for the 3 LHON assays and the coefficient of variance for the 3243, 8344, and 8993 assays was 1.2-52.7, 1.65-5.47, and 0.6-3.5 respectively. Representative pyrograms obtained for a mutated sample from each assay are shown in Figure 2.

\section{Threshold detection of $A 3243 G$ mutation}

The lowest level of detection of the A3243 mutation for the Pyrosequencing, nonfluorescent PCR-RFLP and fluorescent PCR-RFLP assay was determined by generating a standard curve from cloned wild-type and mutated DNA samples that had been mixed to generate samples with the A3243G mutation present at levels of $0 \%, 1 \%, 2.5 \%, 5 \%, 10 \%, 20 \%, 30 \%$, $40 \%, 50 \%, 60 \%, 70 \%, 80 \%, 90 \%$, and $100 \%$. Figure 3 shows the standard curves for the Pyrosequencing and fluorescent PCR-RFLP assays for identical samples analyzed in triplicate. A level of $1 \%$ heteroplasmy was reliably detected with a mean AQ value of 1.73 (standard deviation 0.84). Results from the fluorescent PCR-RFLP show that 5\% heteroplasmy is the limit of detection for this technique. In contrast to the expected linear relationship between observed and expected heteroplasmy levels for the Pyrosequencing assay an apparent quadratic relationship was seen for PCR-RFLP. This may reflect the underrepresentation of heteroplasmy detected because of the formation of heteroduplexes that cannot be cut by the restriction enzyme. Analysis of the same PCR products by agarose gel electrophoresis detected the mutation with a sensitivity of only $20 \%$ (data not shown) which is consistent with other studies (Hancock et al., 2002).

\section{Cost-effectiveness and speed of analysis}

Costings for the Pyrosequencing assays and fluorescent PCR-RFLP using 2004 list prices were comparable to the cost per sample being $£ 1.20$ (GBP) and $£ 1.17$, respectively, excluding system costs and machine maintenance contracts. Af- 
A G3460A Sample $5(100 \%)$

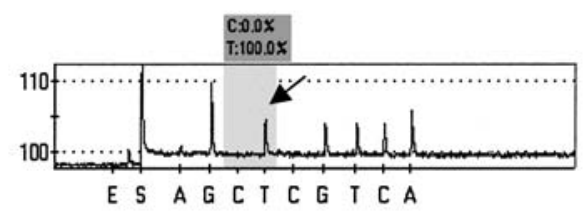

B G11778A Sample $16(100 \%)$

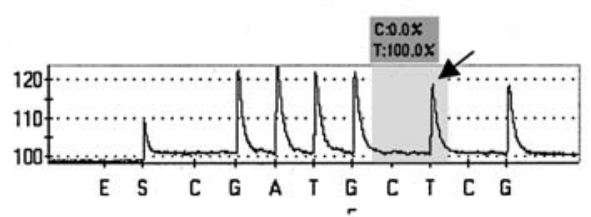

C T14484C Sample 8 (100\%)

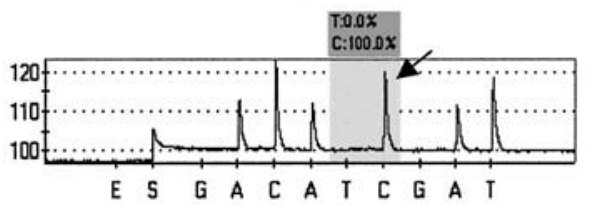

D A3243G Sample $35(12.7 \%)$

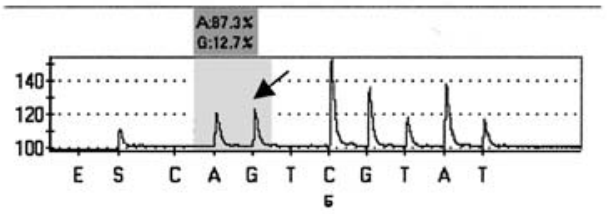

E A8344G Sample 9 (34.9\%)

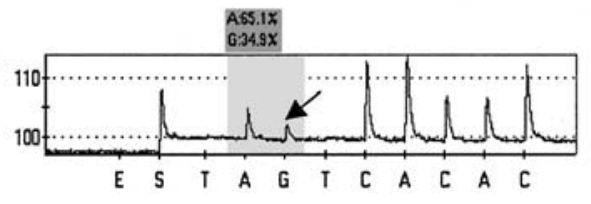

F T8993G Sample $46(100 \%)$

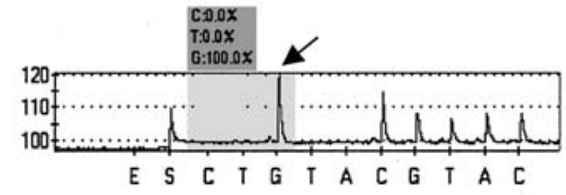

FIG. 2. Representative pyrograms for representative patient samples that are heteroplasmic for (A) G3460A, (B) G11778A, (C) T14484C, (D) A3243G, (E) A8344G, and (F) T8993G. The arrows mark the position corresponding to the mutated base. The boxes show the AQ values obtained for each allele, the value for the mutated allele represents the level of heteroplasmy present in the sample.

ter the PCR, the analysis time is faster using the Pyrosequencing system with a result being available in 30 minutes as compared to 2 hours for fluorescent PCR-RFLP.

\section{DISCUSSION}

Many commonly used molecular biology techniques have been adapted to characterize and quantitate mitochondrial mutations. With the continuing discovery of new mitochondrial mutations and increasing demand for molecular testing, it is important that clinically relevant levels of heteroplasmy can be detected in a reliable and time- and cost-effective manner. It is technically possible to undertake prenatal diagnosis and genetic counselling for mtDNA mutations but predicting the severity and type of symptoms has been hampered by the problems associated with interpreting the level of heteroplasmy. Here, we have examined how Pyrosequencing can be used effectively to genotype and estimate the level of heteroplasmy for six mitochondrial mutations compared to the widely used diagnostic technique PCR-RFLP analysis.

Ninety-five percent of LHON patients harbor one of three mtDNA point mutations that affect genes encoding complex 1 subunits of the respiratory chain; G11778A (Wallace et al., 1988), T14484C (Johns et al., 1992), and G3460A (Huoponen et al., 1991). They differ from other pathogenic mitochondrial mutations because in the majority of pedigrees affected family members are homoplasmic for these mutations (Thornburn and Dahl, 2001). In our study 13 samples were identified as having one of the LHON mutations by PCR-RFLP. The Pyrosequencing assays correctly genotyped these 13 samples and also produced reliable estimates of heteroplasmy with a sensitivity and specificity of $100 \%$. The assay for the G3460A mutation showed the highest level of background signal for normal samples with a mean AQ value for the A allele of $2.88 \%$ (standard deviation 1.62), which precludes the detection of samples with levels of heteroplasmy below $8 \%$. However, because LHON mutations are usually homoplasmic this does not represent a significant problem when using this assay in a diagnostic setting. The assays for G11778A and T14484C were very robust and reproducible and had low background signals for normal samples.

The tRNA gene mutation A3243G was first described by Ciafaloni et al. in a MELAS patient (Ciafaloni et al., 1992). Eighty percent of patients with MELAS have the A3243G mutation, which is the most common mtDNA point mutation detected in approximately 2000 patients with suspected mtDNA disorders (Wong and Senadheera, 1997; Liang and Wang, 1998). The mutation is associated with stroke-like episode and mitochondrial myopathy (MELAS) when present at more than $85 \%$ mutant load but is associated with maternally inherited diabetes mellitus and deafness when present at a lower percentage $(0.1 \%-30 \%)$. Hence, the detection of low-frequency heteroplasmy for this mutation is particularly important because it can be clinically significant. The mutation is usually present in low amounts in tissues that can be sampled in a noninvasive manner (e.g., blood) and it appears that levels of the mutation decrease in blood and increase in muscle over time. Sensitive 
detection methods are therefore important for mutation detection in elderly patients because this can alleviate the need for more invasive muscle biopsy samples to be taken. In our study 10 patients ( 9 peripheral blood samples and 1 muscle biopsy) were found to have the A3243G mutation. The data from the Pyrosequencing assays correlated well with results obtained using PCR-RFLP and the assay had a sensitivity and specificity of $100 \%$ with a cutoff AQ value of $0.39 \%$. The Pyrosequencing assay had very low-level background signals with a mean AQ value for the $\mathrm{G}$ allele of $0.06 \%$ (standard deviation 0.11 ) determined by the analysis of the normal samples. Using samples with known mutation loads it was established that the lowest level of heteroplasmy that could be detected reliably was $1 \%$ that produced a mean AQ value of $1.73 \%$ (standard deviation 0.84) in the Pyrosequencing assay. This threshold level of detection should be adequate for most clinical diagnostic laboratories.

The A8344G mutation in the tRNA Lys gene was first described by Silvestri et al. in association with myoclonic epilepsy with red ragged fibers (Silvestri et al., 1993). The pathogenic MELAS and MERRF mutations both affect the mitochondrial tRNA genes and they have similar effects on the respiratory chain in vitro. As for the MELAS A3243G, there is a correlation between percent heteroplasmy and clinical features in muscle biopsy samples where patients with more than $90 \%$ mutated DNA in muscle are more likely to have deafness, ataxia, and myoclonus than patients who have mutated DNA present at more than $80 \%$ (Chinnery et al., 1997). Unlike the A3243G mutation individuals with A8344G often have similar levels of mutant mtDNA in their blood and muscle. Four patients in this study were found to have the MERRF A8344G mutation present in peripheral blood samples using both Pyrosequencing and PCR-RFLP. The level of heteroplasmy determined by Pyrosequencing assay correlated well to that detected using PCR-RFLP. Again, the Pyrosequencing assay had very low background with the mean AQ value for the $\mathrm{G}$ allele in normal samples being 0.13 (standard deviation 0.5 ) and the assay results were reproducible with a sensitivity and specificity of $100 \%$.
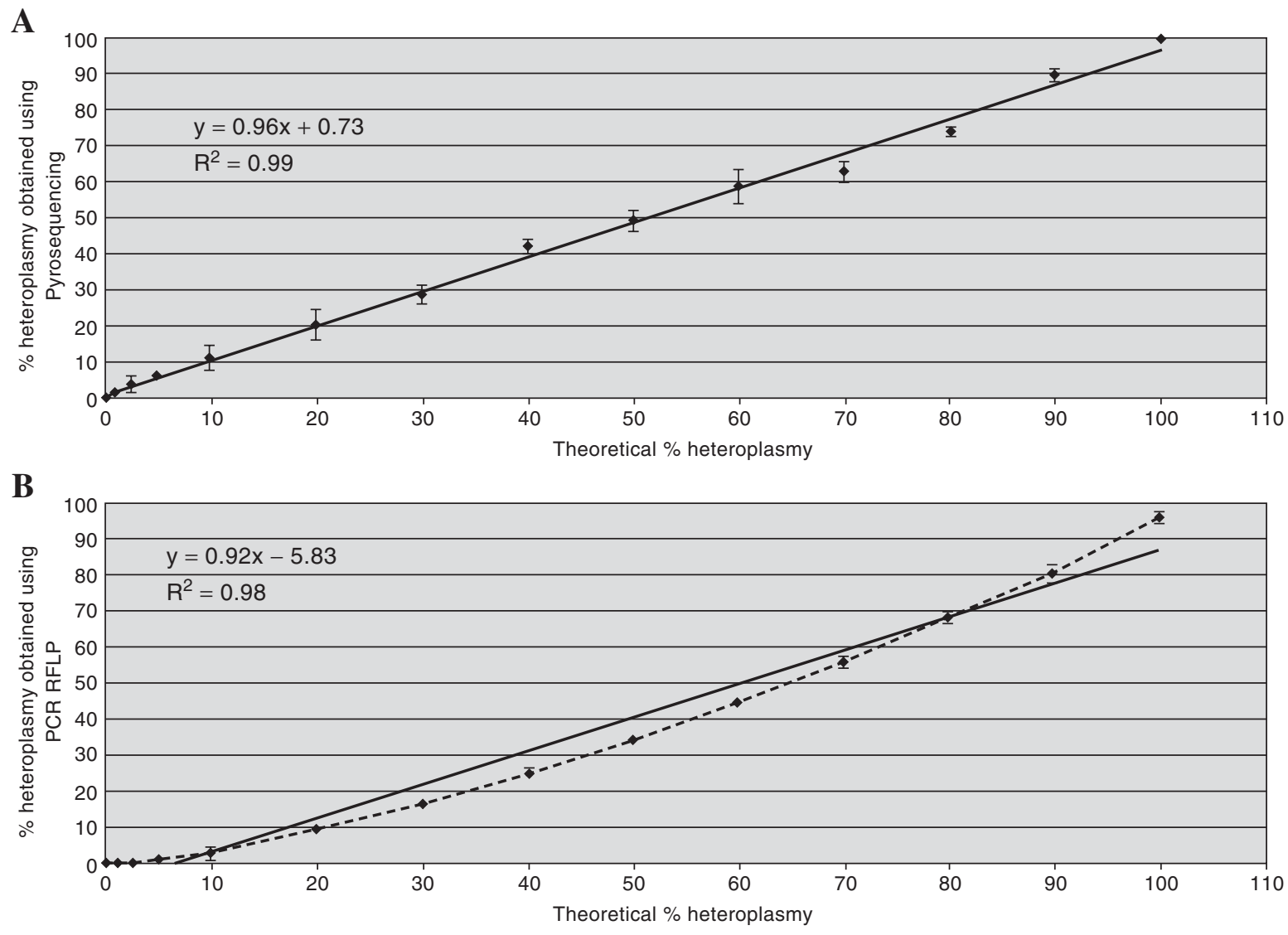

FIG. 3. Standard curves from cloned wild type and mutated DNA samples which have been mixed to generate samples with the A3243G mutation present at levels of $0,1,2.5,5,10,20,30,40,50,60,70,80,90$, and 100\% (x axis). Error bars indicate the standard deviation for triplicate samples. A: Standard curve for the Pyrosequencing assay showing a linear relationship between the percentage heteroplasmy present in the samples and the percentage heteroplasmy detected by the Pyrosequencing assay. The lowest reliable level of A3243G detection was $1 \%$ ( $0.5 \%$ not tested). B: Standard curve for the polymerase chain reaction-restriction fragment length polymorphism (PCR-RFLP) assay showing the linear (solid line) and quadratic (dotted line; $y=$ $\left.0.005 \mathrm{x}^{2}+0.42 \mathrm{x}-0.94, R^{2}=0.99\right)$ relationships between the percentage heteroplasmy present in the samples and the percentage heteroplasmy determined by analysis of the cleaved and uncleaved peak areas after restriction digestion. The lowest reliable level of A3243G detection was $5 \%$. 
The T8993G/C mutation affects the ATP synthase 6 subunit resulting in reduced ATP synthesis and is invariably heteroplasmic. It was first reported in 1990 in a family with NARP syndrome (Holt et al., 1990). The T8993G and T8993C mutations are among the most common mtDNA mutations reported in children and the T8993C mutation is generally considered to be a milder variant (Rahman et al., 1996; Santorelli et al., 1997). Variable clinical expression within families has been reported and two main phenotypes have been identified; NARP syndrome and maternally inherited Leigh syndrome that can be distinguished by different degrees of heteroplasmy of the T8993G mutation. Symptoms usually appear when the mutant load is greater than $60 \%$ with retinal dystrophy related visual loss being the prevalent symptom in the $60 \%-75 \%$ range; NARP syndrome usually occurs between $75 \%$ and $90 \%$ heteroplasmy. The more severe phenotype of Leigh syndrome occurs when levels increase above $95 \%$. The probability of having severe symptoms is low until the mutant load reaches $60 \%-70 \%$ for T8993G and $80 \%-90 \%$ for $\mathrm{T} 8993 \mathrm{C}$ when there is a rapid increase in severity of symptoms with increased mutant load (White et al., 1999).

In this study three patients were found to have the T8993G mutation (two peripheral blood sample and one muscle biopsy), one carried the T8993C mutation and no samples had the G8994A polymorphism. The assay had $100 \%$ sensitivity and specificity when the cutoff level for detection of mutated samples was $0.04 \%$ and the quantitation was reproducible with coefficients of variation (CVs) ranging from 0.6-3.5. The
T8993G/C mutation is usually detected by PCR followed by digestion of the product with HpaII, where a site gain is created by both sequence changes. The polymorphic $\mathrm{G}$ to $\mathrm{A}$ transition at 8994 will abolish this recognition site and therefore patients who have the 8994 polymorphism and the 8993 mutation will be given a false-negative diagnosis using this methodology. The 8994 polymorphism is commonly tested for using the enzyme HaeIII because this site is destroyed by the polymorphism. In patients with the 8994 polymorphism the PCR product is usually sequenced to exclude the 8993 mutation. With Pyrosequencing assays the disease associated genotype and percent heteroplasmy were accurately determined using a single dispensation order. For individuals with the G8994A polymorphism the analysis will fail because of an unrecognized reference pattern as indicated in (Fig. 4). These samples can be rescreened using the dispensation order ACGTCAGCGT (Fig. 4B).

The Pyrosequencing assays provided both accurate genotyping and determination of percent mutation load. The allele frequency quantification function of the SNP software allows automated calling of percent heteroplasmy at the mutated base and a confidence score is given for each sample analyzed (either passed, checked or failed) which alerts the user to the quality of the assay data. The parameters taken into account are the agreement between the observed and expected sequence, the signal-to-noise ratios, and also the peak width. The mutations are presented in sequence context and therefore polymorphic variants will be identified and several

\section{A Sequence to analyze: (C/G/T)GGCCGTAC}

i $c r T$

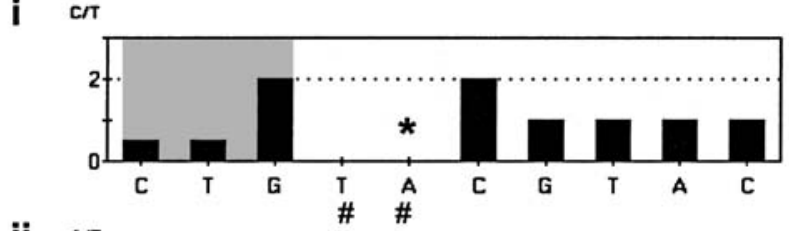

ii or

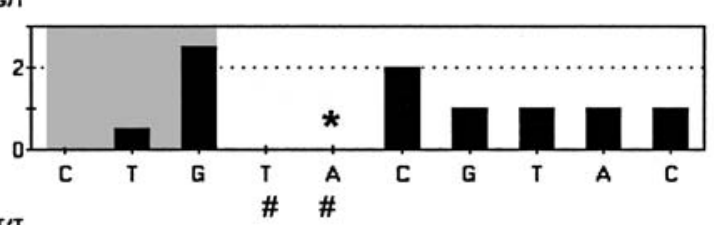

iii $T / T$

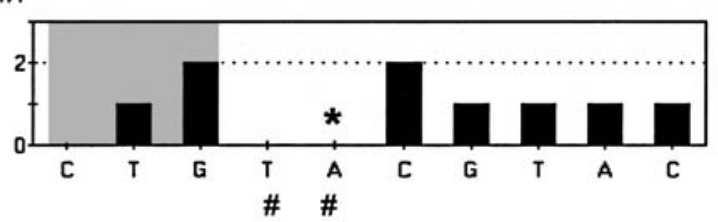

B Sequence to analyze: (C/G/T)AGCCGT

iv

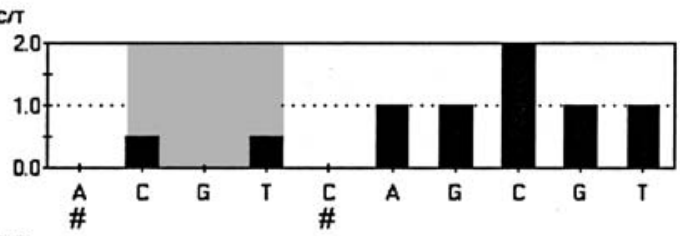

V $\quad 6 / T$

vi
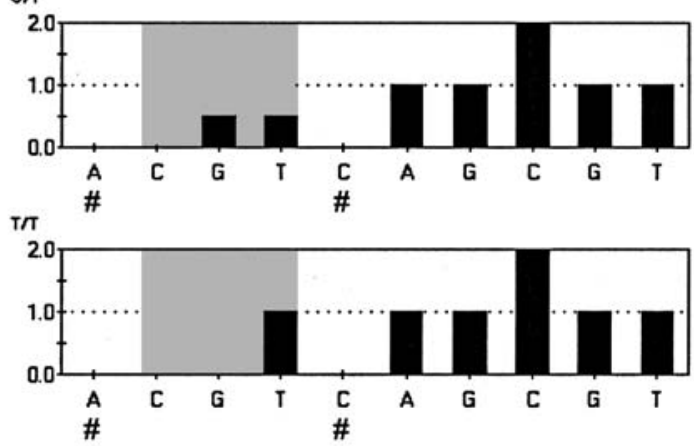

FIG. 4. Pyrosequencing histograms showing results expected for samples with (i) and (iv) $50 \%$ heteroplasmy for T8993C; (ii) and (v) 50\% heteroplasmy for T8993G; (iii) and (vi) no mutation. Reference peaks where no signal is expected are indicated by \#. A: Dispensation order used to detect the T8993G/C mutation. This dispensation order will determine the percentage heteroplasmy and mutation status for samples without G8994A polymorphism. Samples with a G8994A polymorphism will cause an A peak to occur at dispensation 5 (reference peak indicated by *). Because the resulting reference pattern will be unrecognized, the sample will be reported as failed. Therefore, manual examination of all failed samples is necessary to determine whether samples are polymorphic at this position. B: Dispensation order to be used on samples with a known G8994A polymorphism. The percentage heteroplasmy and mutation status can be determined using this assay only when the G8994A polymorphism is present. 
reference peaks are also incorporated into the analysis that add confidence to the data collection. This provides additional benefit compared to PCR-RFLP or techniques that rely upon hybridization where false-positive and -negative results can be obtained in patients with polymorphisms that disrupt the hybridization or restriction enzyme sites or where incomplete restriction enzymes digestion can occur (e.g., Kirby et al., 1998, White et al., 1998). The Pyrosequencing assays were easy to design and optimise and sample processing and use of the machine were straightforward. The incorporation of new and existing mutations into mitochondrial point mutation screening would be relatively undemanding with the advantage that the assays would not have to be designed to include restriction enzyme sites. The platform is very flexible and is amenable to the analysis of either individual mutations in single wells or the analysis of multiple samples for the same mutation within the same run. This is an advantage over PCR-RFLP where different, and sometimes multiple, restriction digests are required for the analysis of each mutation. Pyrosequencing is more sensitive and rapid compared to fluorescent PCR-RFLP with costings for each technique being similar. We conclude therefore that Pyrosequencing is a robust, effective, and efficient means of detecting and quantifying mitochondrial mutations in a clinical laboratory setting.

\section{REFERENCES}

Bai RK, Wong LJ. Detection and quantification of heteroplasmic mutant mitochondrial DNA by real-time amplification refractory mutation system quantitative PCR analysis: A single-step approach. Clin Chem, 50:996-1001, 2004.

Boles RG, Chaudhari D, Soderkvist J, et al. Quantification of mitochondrial DNA heteroplasmy by temporal temperature gradient gel electrophoresis. Clin Chem, 49:198-200, 2003.

Chinnery PF, Howell N, Lightowlers RN, et al. Molecular pathology of MELAS and MERRF. The relationship between mutation load and clinical phenotypes. Brain, 120:1713-1721, 1997.

Ciafaloni E, Ricci E, Shanske S, et al. MELAS: clinical features, biochemistry, and molecular genetics. Ann Neurol, 31:391-398, 1992.

Conley YP, Brockway H, Beatty M, et al. Qualitative and quantitative detection of mitochondrial heteroplasmy in cerebrospinal fluid using denaturing high-performance liquid chromatography. Brain Res Brain Res Protoc, 12:99-103, 2003.

DiMauro S, Schon EA. Nuclear power and mitochondrial disease. Nat Genet, 19:214-215, 1998.

Hancock DK, Schwarz FP, Song F, et al. Design and use of a peptide nucleic acid for detection of the heteroplasmic low-frequency mitochondrial encephalomyopathy, lactic acidosis, and stroke-like episodes (MELAS) mutation in human mitochondrial DNA. Clin Chem, 48:2155-2163, 2002.

He L, Chinnery PF, Durham SE, et al. Detection and quantification of mitochondrial DNA deletions in individual cells by real-time PCR. Nucleic Acids Res, 30:e68, 2002.

Holt IJ, Harding AE, Petty RK, et al. A new mitochondrial disease associated with mitochondrial DNA heteroplasmy. Am J Hum Genet, 46:428-433, 1990.

Huoponen K, Vilkki J, Aula P, et al. A new mtDNA mutation associated with Leber hereditary optic neuroretinopathy. Am J Hum Genet, 48:1147-1153, 1991.
Johns DR, Neufeld MJ, Park RD. An ND-6 mitochondrial DNA mutation associated with Leber hereditary optic neuropathy. Biochem Biophys Res Commun, 187:1551-1557, 1992.

Juvonen V, Huoponen K, Syvanen AC, et al. Quantification of point mutations associated with Leber hereditary optic neuroretinopathy by solid-phase minisequencing. Hum Genet, 93:16-20, 1994.

Kirby DM, Milovac T, Thorburn DR. A false-positive diagnosis for the common MELAS (A3243G) mutation caused by a novel variant (A3426G) in the ND1 gene of mitochondria DNA. Mol Diagn, 3:211-215, 1998.

Lagerström-Fermér M, Olsson C, Forsgren L, et al. Heteroplasmy of the human mtDNA control region remains constant during life. Am J Hum Genet, 68:1299-1301, 2001.

Liang MH, Wong LJ. Yield of mtDNA mutation analysis in 2,000 patients. Am J Med Genet, 77:395-400, 1998.

Macmillan C, Lach B, Shoubridge EA. Variable distribution of mutant mitochondrial DNAs (tRNA(Leu[3243]) in tissues of symptomatic relatives with MELAS: The role of mitotic segregation. Neurology, 43:1586-1590, 1993.

Mashima Y, Nagano M, Funayama T, et al. Rapid quantification of the heteroplasmy of mutant mitochondrial DNAs in Leber's hereditary optic neuropathy using the Invader technology. Clin Biochem, 37:268-276, 2004.

Mashima Y, Saga M, Hiida Y, et al. Quantitative determination of heteroplasmy in Leber's hereditary optic neuropathy by single-strand conformation polymorphism. Invest Ophthalmol, Vis Sci 36:17141720, 1995.

Rahman S, Blok RB, Dahl HH, et al. Leigh syndrome: clinical features and biochemical and DNA abnormalities. Ann Neurol, 39:343-351, 1996.

Ronaghi M, Uhlén M, Nyrén P. A sequencing method based on realtime pyrophosphate. Science, 281:363-365, 1998.

Santorelli FM, Tanji K, Shanske S, et al. Heterogeneous clinical presentation of the mtDNA NARP/T8993G mutation. Neurology, 49:270-273, 1997.

Silvestri G, Ciafaloni E, Santorelli FM, et al. Clinical features associated with the $\mathrm{A} \rightarrow \mathrm{G}$ transition at nucleotide 8344 of mtDNA ("MERRF mutation"). Neurology, 43:1200-1206, 1993.

Suomalainen A, Syvanen AC. Quantitative analysis of human DNA sequences by PCR and solid-phase minisequencing. Mol Biotechnol, 15:123-131, 2000.

Szuhai K, Ouweland J, Dirks R, et al. Simultaneous A8344G heteroplasmy and mitochondrial DNA copy number quantification in myoclonus epilepsy and ragged-red fibers (MERRF) syndrome by a multiplex molecular beacon based real-time fluorescence PCR. Nucleic Acids Res, 29:E13, 2001

Tanno Y, Yoneda M, Tanaka K, et al. Quantitation of heteroplasmy of mitochondrial tRNA(Leu(UUR)) gene using PCR-SSCP. Muscle Nerve, 18:1390-1397, 1995.

Thornburn DR, Dahl H-HM. Mitochondrial disorders: Genetics, counseling, prenatal diagnosis and reproductive options. Am J Med Genet, 106:102-114, 2001.

Tully LA, Parsons TJ, Steighner RJ, et al. A sensitive denaturing gradient-Gel electrophoresis assay reveals a high frequency of heteroplasmy in hypervariable region 1 of the human mtDNA control region. Am J Hum Genet, 67:432-443, 2000.

Urata M, Wada Y, Kim SH, et al. High-sensitivity detection of the A3243G mutation of mitochondrial DNA by a combination of allelespecific PCR and peptide nucleic acid-directed PCR clamping. Clin Chem, 50:2045-2051, 2004.

Wallace DC, Singh G, Lott MT, et al. Mitochondrial DNA mutation associated with Leber's hereditary optic neuropathy. Science, 242:1427-1430, 1988.

Wallace DC. Mitochondrial diseases in man and mouse. Science, 283:1482-1488, 1999. 
White SL, Collins VR, Wolfe R, et al. Genetic counseling and prenatal diagnosis for the mitochondrial DNA mutations at nucleotide 8993. Am J Hum Genet, 65:474-482, 1999.

White SL, Thorburn DR, Christodoulou J, et al. Novel mitochondrial DNA variant that may give a false positive diagnosis for the T8993C mutation. Mol Diagn, 3:113-117, 1998.

Wong LJ, Senadheera D. Direct detection of multiple point mutations in mitochondrial DNA. Clin Chem, 43:1857-1861, 1997.
Address reprint requests to:

Helen E. White, Ph.D.

National Genetics Reference Laboratory (Wessex)

Salisbury District Hospital

Salisbury SP2 8BJ

United Kingdom

E-mail: H.E.White@ soton.ac.uk 